MSC 68-04

\title{
The explicit formula for solution of anomalous diffusion equation in the multi-dimensional space
}

Durdimurod K. Durdiev

Bukhara Branch of the Institute of Mathematics at the Academy of Sciences of the Republic of Uzbekistan, Bukhara, Uzbekistan durdiev65@mail.ru

Elina L. Shishkina

Voronezh State University, Voronezh, Russia

shishkina@amm.vsu.ru

Sergei M. Sitnik

Belgorod State University, Belgorod, Russia

sitnik@bsu.edu.ru

Keywords: Anomalous diffusion; integro-differential equation; Gerasimov-Caputo fractional derivative; Laplace transform; Fourier transform; convolution theorem; explicit solution

Abstract. This paper intends on obtaining the explicit solution of $n$-dimensional anomalous diffusion equation in the infinite domain with non-zero initial condition and vanishing condition at infinity. It is shown that this equation can be derived from the parabolic integro-differential equation with memory in which the kernel is $t^{-\alpha} E_{1-\alpha, 1-\alpha}\left(-t^{1-\alpha}\right), \alpha \in(0,1)$, where $E_{\alpha, \beta}$ is the Mittag-Liffler function. Based on Laplace and Fourier transforms the properties of the Fox Hfunction and convolution theorem, explicit solution for anomalous diffusion equation is obtained.

\section{Contents}

$\begin{array}{lll}1 & \text { Introduction to the problem and its setting } & 1\end{array}$

2 Preliminaries $\quad 3$

3 Explicit solution of the problem (1.1) and (1.2) 6

4 The integro-differential diffusion equation with the Mittag-Leffler function in the kernel

10

5 Conclusions $\quad 11$

\section{Introduction to the problem and its setting}

The processes of transport and aftereffect in disordered inhomogeneous composite media often have kinetics that do not obey the normal (Gaussian) distribution. For this reason, such 
processes are usually called anomalous or non-classical. They are observed experimentally in the study of diffusion in turbulent flows, heat and mass transfer in plasma, filtration of fluids in inhomogeneous porous media, impurity transfer in complex geological formations, charge transfer in amorphous semiconductors, relaxation processes in polymers, hydrodynamics of non-Newtonian fluids, the evolution of complex biological systems, the transfer of information resources to global communication networks and many other phenomena.

The rapid development of fractional differential equations was largely due to the discovered practical applications of fractional calculus, primarily in the physics of complex inhomogeneous media. It turned out that fractional differential equations are ideally suited for modeling anomalous processes occurring in systems with a fractal structure or having a power-law memory. As a result, integro-differentiation of fractional order began to develop as a powerful modern apparatus of mathematical modeling, including both analytical and numerical methods for studying fractional differential models.

The most common and studied fractional differential models are models of various anomalous diffusion-type transfer processes, the kinetics of which are well described (at least asymptotically) by power laws with fractional exponents. The value of this exponent, depending on the standard deviation of the positions of the diffusing particles (if its final value exists) versus time, allows us to divide the processes of anomalous diffusion into subdiffusion ones and superdiffusion ones. With subdiffusion, the particle transfer intensity is lower than with classical diffusion. Such processes are usually observed in systems with memory. The superdiffusion phenomenon has a higher particle transfer rate than classical diffusion and is often observed in media with a fractal structure. In systems with both spatial and temporal non locality, both sub- and superdiffusion processes can occur [1]. Examples of fractional differential equations of the diffusion type are the properly equations of subdiffusion and superdiffusion [2 6], diffusion-wave equations [7-11], fractional differential equations of Fokker-Plann [12 16], advection-dispersion [17 20], reactiondiffusion [21-23] and a number of others.

The solvability of Cauchy problems and initial-boundary value problems for various types of linear fractional differential equations of diffusion type were investigated in the works of A.A. Kilbas, S.M. Sitnik, A.N. Kochubey, Yu. Luchko, R. Gorenflo, F. Mainardi, M. M. Meerschaert, G. Pagnini, J.J. Trujillo and many others [24-34]. To construct a solution of linear fractional differential equations of diffusion type, various methods and algorithms based on the Green's function, Fourier, Laplace, and Mellin integral transforms, a generalization of the method of separation of variables, reduction to Volterra-type integral equations, and several others were proposed. At the same time, there are practically no methods for obtaining analytical solutions of fractional differential equations describing anomalous diffusion processes.

In this paper, we consider the following $n$-dimensional integro-differential equation of the anomalously diffusive transport of solute in heterogeneous porous media [35]

$$
u_{t}(x, t)+{ }_{0}^{C} D_{t}^{\alpha} u-\Delta u(x, t)=f(x, t),
$$

which satisfies the initial and boundary conditions

$$
u(x, 0)=g(x), \quad \lim _{|x| \rightarrow \infty}(u, \nabla u)(x, t)=0, \quad t>0, \quad x=\left(x_{1}, x_{2}, \ldots, x_{n}\right) \in \mathbb{R}^{n},
$$


where the Gerasimov-Caputo fractional differential operator ${ }_{0}^{C} D_{t}^{\alpha}$ is defined by [25]

$$
{ }_{0}^{C} D_{t}^{\alpha} g(t):={ }_{0} I_{t}^{1-\alpha} f^{\prime}(t)=\frac{1}{\Gamma(1-\alpha)} \int_{0}^{t} \frac{f^{\prime}(\tau)}{(t-\tau)^{\alpha}} d \tau,
$$

where

$$
{ }_{0} I_{t}^{\alpha} g(t):=\frac{1}{\Gamma(\alpha)} \int_{0}^{t} \frac{f(\tau)}{(t-\tau)^{1-\alpha}} d \tau
$$

$\Delta$ is the $n$-dimensional Laplace operator with respect to $x$ and $\nabla=\left(\frac{\partial}{\partial x_{1}}, \ldots, \frac{\partial}{\partial x_{n}}\right)$.

\section{Preliminaries}

In this section, we present well known definitions and lemmas that will be used for proof of main results.

The Mittag-Leffler functions $E_{\alpha}(z)$ and $E_{\alpha, \beta}(z)$ are defined by the following series:

$$
E_{\alpha}(z):=\sum_{n=0}^{\infty} \frac{z^{n}}{\Gamma(\alpha n+1)}=: E_{\alpha, 1}(z)
$$

and

$$
E_{\alpha, \beta}(z):=\sum_{n=0}^{\infty} \frac{z^{n}}{\Gamma(\alpha n+\beta)},
$$

respectively, where $\alpha, z, \rho \in \mathbb{C} ; \mathfrak{R}(\alpha)>0$. These functions are natural extensions of the exponential, hyperbolic and trigonometric functions, since

$$
E_{1}(z)=e^{z}, \quad E_{2}\left(z^{2}\right)=\cosh z, \quad E_{2}\left(-z^{2}\right)=\cos z, \quad E_{1,2}(z)=\frac{e^{z}-1}{z}, \quad E_{2,2}\left(z^{2}\right)=\frac{\sinh z}{z} .
$$

The three-parameter Mittag-Leffler function or Prabhakar function is [36]:

$$
E_{\alpha, \beta}^{\gamma}:=\sum_{n=0}^{\infty} \frac{(\gamma)_{n}}{\Gamma(\alpha n+\beta)} \frac{z^{n}}{n !}
$$

where $\alpha, \beta, \gamma, z \in \mathbb{C}$, and $(\gamma)_{n}$ denotes the Pochammer symbol or the shifted factorial defined by

$$
(\gamma)_{0}=1, \quad(\gamma)_{n}=\gamma(\gamma+1) \ldots(\gamma+n-1), \quad \gamma \neq 0
$$

Also we can write

$$
(\gamma)_{n}=\frac{\Gamma(\gamma+n)}{\Gamma(\gamma)}
$$


where $\Gamma(\gamma)$ is the Gamma function. We have following special cases: $E_{\alpha, \beta}^{1}(z)=E_{\alpha, \beta}(z)$ and $E_{\alpha, 1}^{1}=E_{\alpha}(z)$.

Recall that the function (2.1) can be rewritten in terms of the Fox H-function as [36, 37]:

$$
E_{\alpha, \beta}^{\gamma}(z)=\frac{1}{\Gamma(\gamma)} H_{1,2}^{1,1}\left[-z \mid \begin{array}{l}
(1-\gamma, 1) \\
(0,1),(1-\beta, \alpha)
\end{array}\right]
$$

We define the integral operator $\mathcal{E}_{\alpha, \beta, \omega ; a+}^{\gamma}$ as follows [36, 38]:

$$
\left(\mathcal{E}_{\alpha, \beta, \omega ; a+}^{\gamma} \varphi\right)(t):=\left(t^{\beta-1} E_{\alpha, \beta}^{\gamma}\left(\omega t^{\alpha}\right)\right) * \varphi(t)=\int_{a}^{t}(t-\tau)^{\beta-1} E_{\alpha, \beta}^{\gamma}\left(\omega(t-\tau)^{\alpha}\right) \varphi(\tau) d \tau
$$

Note the integral operator (2.2) is nowadays known in literature as Prabhakar fractional integral.

Lemma 1. The following Laplace transform of a three-parameter Mittag-Leffler function is true [36, 39]:

$$
L\left[t^{\beta-1} E_{\alpha, \beta}^{\gamma}\left( \pm \omega t^{\alpha}\right)\right](s)=\int_{0}^{\infty} e^{-s t} t^{\beta-1} E_{\alpha, \beta}^{\gamma}\left( \pm \omega t^{\alpha}\right) d t=\frac{s^{\alpha \gamma-\beta}}{\left(s^{\alpha} \mp \omega\right)^{\gamma}},
$$

where $\left|\omega / s^{\alpha}\right|<1$.

Lemma 2. The Laplace transform of $e^{-\lambda t} t^{\beta-1} E_{\alpha, \beta}^{\gamma}\left( \pm \omega t^{\alpha}\right)$ is given by the following formula 39]:

$$
L\left[e^{-\lambda t} t^{\beta-1} E_{\alpha \beta}^{\gamma}\left( \pm \omega t^{\alpha}\right)\right](s)=\frac{(s+\lambda)^{\alpha \gamma-\beta}}{\left((s+\lambda)^{\alpha} \mp \omega\right)^{\gamma}},
$$

where $\lambda \geq 0,\left|\omega /(s+\lambda)^{\alpha}\right|<1$.

In the case $\lambda=0$, Lemma 2 coincides with Lemma 1.

Lemma 3. For arbitrary $\alpha>0, \beta$ is an arbitrary complex number, $\mu>0$ and $a \in \mathbb{R}$, the following formula is valid [20]:

$$
\int_{\mathbb{R}^{n}} e^{i \xi \cdot x} E_{\alpha, \beta}^{(n)}\left(-a|\xi|^{\mu}\right) d \xi=(2 \pi)^{n / 2}|x|^{1-n / 2} \int_{0}^{\infty}|\xi|^{n / 2} E_{\alpha, \beta}^{(n)}\left(-a|\xi|^{\mu}\right) J_{\frac{n}{2}-1}(|x||\xi|) d|\xi| .
$$

Here $J_{\frac{n}{2}-1}(\cdot)$ is a Bessel function and $E_{\alpha, \beta}^{(n)}(z)$ denotes $n$-th derivatives of the Mittag-Leffler function. $n$-th derivatives of the Mittag-Leffler function can be expressed in terms of the Fox H-function as

$$
E_{\alpha, \beta}^{(n)}(z)=H_{1,2}^{1,1}\left[-z \mid \begin{array}{l}
(-n, 1) \\
(0,1),(1-(\alpha n+\beta), \alpha)
\end{array}\right] .
$$

Lemma 4. If $\{k(t), r(t)\} \in L_{1}[0, T]$ for a fixed $T>0$ and $k(t), r(t)$ are connected by the integral equation

$$
r(t)=k(t)+\int_{0}^{t} k(t-\tau) r(\tau) d \tau, \quad t \in[0, T]
$$


then the solution of the integral equation

$$
\varphi(t)=\int_{0}^{t} k(t-\tau) \varphi(\tau) d \tau+f(t), \quad f(t) \in L_{1}[0, T]
$$

is expressed by formula

$$
\varphi(t)=\int_{0}^{t} r(t-\tau) f(\tau) d \tau+f(t) .
$$

Although the assertion of Lemma 4 is well known, we give a original proof.

Proof of Lemma 4. Let the equality (2.3) be satisfied. Then from (2.3) we have

$$
k(t)=r(t)-\int_{0}^{t} r(t-\tau) k(\tau) d \tau .
$$

Taking into account this relation, from (2) we obtain the following chain of equalities:

$$
\begin{aligned}
& \varphi(t)=\int_{0}^{t}\left[r(t-\tau)-\int_{0}^{t-\tau} r(t-\tau-\alpha) k(\alpha) d \alpha\right] \varphi(\tau) d \tau+f(t)=\int_{0}^{t} r(t-\tau) \varphi(\tau) d \tau- \\
& -\int_{0}^{t}\left(\int_{0}^{t-\tau} r(t-\tau-\alpha) k(\alpha) d \alpha\right) \varphi(\tau) d \tau+f(t)=\int_{0}^{t} r(\tau) \varphi(t-\tau) d \tau- \\
& -\int_{0}^{t}\left(\int_{0}^{t-\tau} r(\alpha) k(t-\tau-\alpha) d \alpha\right) \varphi(\tau) d \tau+f(t)=\int_{0}^{t} r(\tau) \varphi(t-\tau) d \tau- \\
& -\int_{0}^{t}\left(\int_{0}^{\tau} r(\alpha) k(\tau-\alpha) d \alpha\right) \varphi(t-\tau) d \tau+f(t)=\int_{0}^{t} r(\tau) \varphi(t-\tau) d \tau- \\
& -\int_{0}^{t} r(\alpha)\left(\int_{\alpha}^{t} k(\tau-\alpha) \varphi(t-\tau) d \tau\right) d \alpha+f(t)= \\
& =\int_{0}^{t} r(\tau)\left[\varphi(t-\tau)-\int_{0}^{t-\tau} k(\tau) \varphi(t-\tau-\alpha) d \alpha\right] d \tau+f(t) .
\end{aligned}
$$

According to (2.4), the expression in the square brackets of the right side in the last equalities is equal to $f(t)$. Therefore, we get $(2.5)$. 
Now we show the assertion of Lemma 4 is true in the opposite direction. Indeed, from (2.5) on based of (2.3), we obtain

$$
\begin{gathered}
\varphi(t)=\int_{0}^{t}\left[k(t-\tau)+\int_{0}^{t-\tau} k(t-\tau-\alpha) r(\alpha) d \alpha\right] f(\tau) d \tau+f(t)= \\
=\int_{0}^{t} k(t-\tau) f(\tau) d \tau+\int_{0}^{t}\left(\int_{0}^{t-\tau} k(t-\tau-\alpha) r(\alpha) d \alpha\right) f(\tau) d \tau+f(t)= \\
=\int_{0}^{t} k(\tau)\left[f(t-\tau)+\int_{0}^{t-\tau} r(\alpha) f(t-\tau-\alpha) d \alpha\right] d \tau+f(t) .
\end{gathered}
$$

From this, in view of (2.5), we obtain (2.3).

\section{Explicit solution of the problem (1.1) and (1.2)}

Let

$$
\begin{gathered}
\tilde{f}(\xi, t):=\int_{\mathbb{R}^{n}} f(x, t) e^{-i \xi \cdot x} d x, \\
x \in \mathbb{R}^{n}, \quad \xi \in \mathbb{R}^{n}, \quad \xi \cdot x=\sum_{j=1}^{n} \xi_{i} \cdot x_{i}, \quad d x=d x_{1} d x_{2} \ldots d x_{n}
\end{gathered}
$$

is the Fourier transform of $f(x, t)$ with respect to the spatial variable $x$ and

$$
\widetilde{g}(\xi):=\int_{\mathbb{R}^{n}} g(x) e^{-i \xi \cdot x} d x
$$

The Laplace transform of a function $u(x, t)$ with respect to the variable $t$ is given by

$$
\hat{u}(x, s)=\int_{0}^{\infty} e^{-s t} u(x, t) d t, \quad t>0
$$

The unknown function $u(x, t)$ is required to be sufficiently well behaved to be treated with its derivatives $u_{t}(x, t), u_{x_{i} x_{i}}(x, t), i=1, \ldots, n$ by technique of Laplace (in $t$ ) and Fourier (in $x$ ) transforms. The given functions $f(x, t)$ and $g(x)$ are also assumed to have such properties.

Theorem 1. The explicit solution of the problem (1.1) and (1.2) can be expressed by formula

$$
u(x, t)=\frac{1}{(2 \pi)^{n}} \int_{\mathbb{R}^{n}} \sum_{j=0}^{\infty}(-1)^{j}\left(\mathcal{E}_{1,(1-\alpha) j+1,-|\xi|^{2} ; 0+}^{j+1} \tilde{f}\right)(\xi, \tau) e^{i \xi \cdot x} d \xi+
$$




$$
+\int_{\mathbb{R}^{n}} G(x-\xi, t) g(\xi) d \xi, \quad d \xi=d \xi_{1} d \xi_{2} \ldots d \xi_{n}
$$

where the Green function $G(x, t)$ is given by

$$
\begin{gathered}
G(x, t)=\frac{1}{(2 \pi)^{n / 2}|x|^{n}} \sum_{j=0}^{\infty} \frac{\left(-t^{1-\alpha}\right)^{j}}{j !} \times \\
\times\left\{H_{2,0}^{1,2}\left[\frac{|x|^{2}}{2 t^{1 / 2}} \mid \begin{array}{c}
(1+(1-\alpha) j, 1 / 2) \\
(n / 2,1 / 2),(2+j, 1 / 2)
\end{array}\right]+H_{2,0}^{1,2}\left[\frac{|x|^{2}}{2 t^{1 / 2}} \mid \begin{array}{c}
(1+(1-\alpha) j, 1 / 2) \\
(n / 2,1 / 2),(1+j, 1 / 2)
\end{array}\right]\right\},
\end{gathered}
$$

where $x \in \mathbb{R}^{n}, \xi \in \mathbb{R}^{n}$.

Proof. Let $F\{u(x, t)\}:=\tilde{u}(\xi, t)$ be the Fourier transform of $u(x, t)$ with respect to variable $x$, and $L\{u(x, t)\}:=\hat{u}(x, s)$ be the Laplace transform of $u(x, t)$ with respect to variable $t$. In sequence, applying to the equation (1.1) the Laplace transform with respect to the time variable $t$ and the Fourier transform with respect to the spatial variable $x$, we obtain the following equation:

$$
s \hat{\tilde{u}}(\xi, s)-\tilde{u}(\xi, 0)+s^{\alpha-1}[s \hat{\tilde{u}}-\tilde{u}(\xi, 0)]=-|\xi|^{2} \hat{\tilde{u}}(\xi, s)+\hat{\tilde{f}}(\xi, s), \xi \in \mathbb{R}^{n} .
$$

Taking into account the initial condition (1.2), this equation yields

$$
\hat{\tilde{u}}(\xi, s)=\frac{1}{s+s^{\alpha}+|\xi|^{2}} \hat{\tilde{f}}(\xi, s)+\frac{1+s^{\alpha-1}}{s+s^{\alpha}+|\xi|^{2}} \tilde{g}(\xi) .
$$

First, we calculate the inverse Laplace and Fourier transforms of the first term on the right side of (3.2). It may be performed by using the equality

$$
\frac{1}{s+s^{\alpha}+|\xi|^{2}}=\frac{s^{-\alpha}}{s^{1-\alpha}+1} \cdot \frac{1}{1+\frac{|\xi|^{2} s^{-\alpha}}{s^{1-\alpha}+1}}
$$

and expanding the third factor on the right of this equation into an infinitely decreasing geometric series:

$$
\frac{1}{1+\frac{|\xi|^{2} s^{-\alpha}}{s^{1-\alpha}+1}}=\sum_{n=0}^{\infty}\left(-|\xi|^{2}\right)^{n} \frac{s^{-\alpha n}}{\left(s^{1-\alpha}+1\right)^{n}}
$$

for $\left|\frac{|\xi|^{2} s^{-\alpha}}{s^{1-\alpha}+1}\right|<1$.

In view of (3.3) from last equality we have

$$
\frac{1}{s+s^{\alpha}+|\xi|^{2}}=\sum_{n=0}^{\infty}\left(-|\xi|^{2}\right)^{n} \frac{s^{-\alpha(n+1)}}{\left(s^{1-\alpha}+1\right)^{n+1}} .
$$

Then, according to Lemma 1, the first term of equation (3.2) can be expressed as

$$
\frac{1}{s+s^{\alpha-1}+|\xi|^{2}} \hat{\tilde{f}}(\xi, s)=L\left[\sum_{n=0}^{\infty}\left(-|\xi|^{2}\right)^{n} t^{n} E_{1-\alpha, n+1}^{n+1}\left(-t^{1-\alpha}\right)\right] L[\tilde{f}(\xi, t)] .
$$


We now transform the second term on the right side of (3.2). For this we note

$$
\begin{gathered}
\frac{1+s^{\alpha-1}}{s+s^{\alpha-1}+|\xi|^{2}}=\left(1+s^{\alpha-1}\right) \cdot \frac{s^{-\alpha}}{s^{1-\alpha}+1} \cdot \frac{1}{1+\frac{|\xi|^{2} s^{-\alpha}}{s^{1-\alpha}+1}}= \\
=\frac{s^{-\alpha}+s^{-1}}{s^{1-\alpha}+1} \sum_{n=0}^{\infty}\left(-|\xi|^{2}\right)^{n} \frac{s^{-\alpha n}}{\left(s^{1-\alpha}+1\right)^{n}}= \\
=\sum_{n=0}^{\infty}\left(-|\xi|^{2}\right)^{n} \frac{s^{-\alpha(n+1)}}{\left(s^{1-\alpha}+1\right)^{n+1}}+\sum_{n=0}^{\infty}\left(-|\xi|^{2}\right)^{n} \frac{s^{-\alpha n-1}}{\left(s^{1-\alpha}+1\right)^{n+1}} .
\end{gathered}
$$

In view of last relations, applying Lemma 1 to the second term of (3.2), we obtain

$$
\frac{1+s^{\alpha-1}}{s+s^{\alpha-1}+|\xi|^{2}} \tilde{g}(\xi)=L\left[\sum_{0}^{\infty}\left(-|\xi|^{2}\right)^{n} t^{n}\left(E_{1-\alpha, n+1}^{n}\left(-t^{1-\alpha}\right)+E_{1-\alpha, n+1}^{n+1}\left(-t^{1-\alpha}\right)\right] \cdot \tilde{g}(\xi) .\right.
$$

Further, in accordance with the Mittag-Leffler function definition (2.1), from equation (3.4) we get

$$
\begin{aligned}
& \sum_{n=0}^{\infty}\left(-|\xi|^{2}\right)^{n} t^{n}\left(E_{1-\alpha, n+1}^{n}\left(-t^{1-\alpha}\right)=\sum_{n=0}^{\infty}\left(-|\xi|^{2}\right)^{n} t^{n} \sum_{j=0}^{\infty} \frac{(n+1)_{j}}{\Gamma((1-\alpha) j+n+1)} \cdot \frac{\left(-t^{1-\alpha}\right)^{j}}{j !}=\right. \\
& =\sum_{n=0}^{\infty} \sum_{j=0}^{\infty}\left(-t^{1-\alpha}\right)^{j} \frac{(j+1)_{n}}{\Gamma((1-\alpha) j+n+1)} \frac{\left(-|\xi|^{2} t\right)^{n}}{n !}=\sum_{j=0}^{\infty}\left(-t^{1-\alpha}\right)^{j} E_{1,(1-\alpha) j+1}^{j+1}\left(-|\xi|^{2} t\right) .
\end{aligned}
$$

By virtue of this fact we continue converting of the right side of (3.4) as

$$
\begin{gathered}
L\left[\sum_{n=0}^{\infty}\left(-|\xi|^{2}\right)^{n} t^{n} E_{1-\alpha, n+1}^{n+1}\left(-t^{1-\alpha}\right)\right] L[\tilde{f}(\xi, t)]=L\left[\sum_{j=0}^{\infty}\left(-t^{1-\alpha}\right)^{j} E_{1,(1-\alpha) j+1}^{j+1}\left(-|\xi|^{2} t\right)\right] \times \\
\times L[\tilde{f}(\xi, t)]=L\left[\left(\sum_{j=0}^{\infty}\left(-t^{1-\alpha}\right)^{j} E_{1,(1-\alpha) j+1}^{j+1}\left(-|\xi|^{2} t\right)\right) * \tilde{f}(\xi, t)\right] .
\end{gathered}
$$

Taking into consideration the convolution property of the Laplace transform and the definition of integral operator $\mathcal{E}_{\alpha, \beta, \omega ; a+}^{\gamma} \varphi$ by (2.2), the inverse transform of the first term in equation (3.2) can be obtained as follows:

$$
L^{-1}\left[\frac{1}{s+s^{\alpha-1}+|\xi|^{2}} L[\tilde{f}(\xi, t)](s)\right]=\sum_{j=0}^{\infty}(-1)^{j}\left(\mathcal{E}_{1,(1-\alpha) j+1,-|\xi|^{2} ; 0+}^{j+1} \tilde{f}\right)(\xi, \tau) .
$$

Analogically, the inverse Laplace transform of the second term in equation (3.2) by virtue of (3.5) can be expressed as

$$
L^{-1}\left[\frac{1+s^{\alpha-1}}{s+s^{\alpha-1}+|\xi|^{2}} \tilde{g}(\xi)\right]=\sum_{n=0}^{\infty}\left(-|\xi|^{2}\right)^{n} t^{n}\left(E_{1-\alpha, n+1}^{n}\left(-t^{1-\alpha}\right)+E_{1-\alpha, n+1}^{n+1}\left(-t^{1-\alpha}\right)\right) \tilde{g}(\xi)=
$$




$$
=\sum_{j=0}^{\infty}\left(-t^{1-\alpha}\right)^{j}\left(E_{1,(1-\alpha) j+1}^{j}\left(-|\xi|^{2} t\right)+E_{1,(1-\alpha) j+1}^{j+1}\left(-|\xi|^{2} t\right)\right) \tilde{g}(\xi) .
$$

Considering the relationship between the generalized Mittag-Leffler function and the Fox-H function, the last equality can be rewritten in the form [37]:

$$
\begin{gathered}
L^{-1}\left[\frac{1+s^{\alpha-1}}{s+s^{\alpha-1}+|\xi|^{2}} \tilde{g}(\xi)\right]=\sum_{j=0}^{\infty}(-1)^{j} t^{(1-\alpha)^{j} \times} \\
\times\left(\frac{1}{\Gamma(j)} H_{1,2}^{1,1}\left[\left.|\xi|^{2} t\right|_{(0,1),(-(1-\alpha) j, 1)} ^{(1-j, 1)}\right]+\frac{1}{\Gamma(j+1)} H_{1,2}^{1,1}\left[\left.|\xi|^{2} t\right|_{(0,1),(-(1-\alpha) j, 1)} ^{(-j, 1)}\right]\right) \tilde{g}(\xi)= \\
=\sum_{j=0}^{\infty} \frac{(-1)^{j}}{j !} t^{(1-\alpha)^{j}}\left(j H_{1,2}^{1,1}\left[\left.|\xi|^{2} t\right|_{(0,1),(-(1-\alpha) j, 1)} ^{(-j, 1)}\right]+H_{1,2}^{1,1}\left[|\xi|^{2} t \mid \begin{array}{l}
(-j, 1) \\
(0,1),(-(1-\alpha) j, 1)
\end{array}\right]\right) \tilde{g}(\xi) .
\end{gathered}
$$

We introduce the following notations:

$$
\tilde{h}_{0 j}(\xi, t)=H_{1,2}^{1,1}\left[|\xi|^{2} t \mid \begin{array}{l}
(1-j, 1) \\
(0,1),(-(1-\alpha) j, 1)
\end{array}\right], \quad \tilde{h}_{1 j}(\xi, t)=H_{1,2}^{1,1}\left[|\xi|^{2} t \mid \begin{array}{l}
(-j, 1) \\
(0,1),(-(1-\alpha) j, 1)
\end{array}\right]
$$

and

$$
\tilde{G}(\xi, t)=\sum_{j=0}^{\infty} \frac{\left(-t^{1-\alpha}\right)^{j}}{j !}\left[j \tilde{h}_{0 j}(\xi, t)+\tilde{h}_{1 j}(\xi, t)\right] .
$$

Applying the inverse transform $F^{-1}$ to equations (3.8), we obtain

$$
\begin{aligned}
& h_{0 j}(x, t)=\frac{1}{(2 \pi)^{n}} \int_{\mathbb{R}^{n}} H_{1,2}^{1,1}\left[|\xi|^{2} t \mid \begin{array}{l}
(1-j, 1) \\
(0,1),(-(1-\alpha) j, 1)
\end{array}\right] e^{i \xi \cdot x} d \xi, \\
& h_{1 j}(x, t)=\frac{1}{(2 \pi)^{n}} \int_{\mathbb{R}^{n}} H_{1,2}^{1,1}\left[|\xi|^{2} t \mid \begin{array}{l}
(-j, 1) \\
(0,1),(-(1-\alpha) j, 1)
\end{array}\right] e^{i \xi \cdot x} d \xi .
\end{aligned}
$$

Using Lemma 3, we obtain the following results from equations (3.10) and (3.11)

$$
\begin{aligned}
& h_{0 j}(x, t)=\frac{1}{(2 \pi)^{n / 2}}|x|^{1-\frac{n}{2}} \int_{0}^{\infty}|\xi|^{n / 2} H_{1,2}^{1,1}\left[\left.|\xi|^{2} t\right|_{\left(\begin{array}{l}
(1-j, 1) \\
(0,1),(-(1-\alpha) j, 1)
\end{array}\right]} \mathfrak{J}_{\frac{n}{2}-1}(|x||\xi|) d|\xi|,\right. \\
& h_{1 j}(x, t)=\frac{1}{(2 \pi)^{n / 2}}|x|^{1-\frac{n}{2}} \int_{0}^{\infty}|\xi|^{n / 2} H_{1,2}^{1,1}\left[\left.|\xi|^{2} t\right|_{\left(\begin{array}{l}
(-j, 1) \\
(0,1),(-(1-\alpha) j, 1)
\end{array}\right]} \mathfrak{J}_{\frac{n}{2}-1}(|x||\xi|) d|\xi| .\right.
\end{aligned}
$$

Taking into account a Hankel transform and the properties Fox-H function [37,41, last two equations can be written as

$$
h_{0 j}(x, t)=\frac{1}{(2 \pi)^{n / 2}}|x|^{n} H_{2,0}^{1,2}\left[\frac{|x|^{2}}{2 t^{1 / 2}} \mid \begin{array}{c}
(1+(1-\alpha) j, 1 / 2) \\
(n / 2,1 / 2),(2+j, 1 / 2)
\end{array}\right],
$$




$$
h_{1 j}(x, t)=\frac{1}{(2 \pi)^{n / 2}}|x|^{n} H_{2,0}^{1,2}\left[\frac{|x|^{2}}{2 t^{1 / 2}} \mid \begin{array}{c}
(1+(1-\alpha) j, 1 / 2) \\
(n / 2,1 / 2),(1+j, 1 / 2)
\end{array}\right] .
$$

Computing the inverse transform of equation (3.9) and substituting into resulting equality formulas (3.12), (3.13), we get

$$
\begin{gathered}
G(x, t)=\frac{1}{(2 \pi)^{n / 2}|x|^{n}} \sum_{j=0}^{\infty} \frac{\left(-t^{1-\alpha}\right)^{j}}{j !}\left\{H_{2,0}^{1,2}\left[\frac{|x|^{2}}{2 t^{1 / 2}} \mid \begin{array}{c}
(1+(1-\alpha) j, 1 / 2) \\
(n / 2,1 / 2),(2+j, 1 / 2)
\end{array}\right]+\right. \\
\left.+H_{2,0}^{1,2}\left[\frac{|x|^{2}}{2 t^{1 / 2}} \mid \begin{array}{c}
(1+(1-\alpha) j, 1 / 2) \\
(n / 2,1 / 2),(1+j, 1 / 2)
\end{array}\right]\right\} .
\end{gathered}
$$

Continuing to convert the equality (3.7) we can write formally

$$
L^{-1}\left[\frac{1+s^{\alpha-1}}{s+s^{\alpha-1}+|\xi|^{2}} \tilde{g}(\xi)\right]=L^{-1}[F[G(x, s)](\xi) \tilde{g}(\xi)]
$$

In view of (3.6) and (3.14), applying an inverse Laplace transform to equation (3.2) we finally obtain

$$
\tilde{u}(\xi, t)=\sum_{j=0}^{\infty}(-1)^{j}\left(\mathcal{E}_{1,(1-\alpha) j+1,-|\xi|^{2} ; 0+}^{j+1} \tilde{f}\right)(\xi, \tau)+F[G(x, t)](\xi) g(\xi) .
$$

To equation (3.15) can be further applied inverse Fourier transform and Fourier convolution property in sequence. Accordingly, the Theorem 1 is proven.

\section{The integro-differential diffusion equation with the Mittag-Leffler function in the kernel}

In this section we show equivalence of one integro-differential diffusion equation with the Mittag-Leffler function in the kernel to the anomalous diffusion equation.

Theorem 2. The integro-differential diffusion equation

$$
u_{t}-\triangle u+\int_{0}^{t} k(t-\tau) \triangle u(x, \tau) d \tau=0, x \in \mathbb{R}^{n}, t>0
$$

with memory

$$
k(t)=t^{-\alpha} E_{1-\alpha, 1-\alpha}\left(-t^{1-\alpha}\right), \alpha \in(0,1),
$$

is equivalent to the time-fractional diffusion equation

$$
u_{t}+{ }_{0}^{C} D_{t}^{\alpha} u-\triangle u(x, t)=0 .
$$


Proof. Considering equation (4.1) as the Volterra integral equation of the second kind with respect to $\triangle u$ for fixed $x$ and applying Lemma 4 , we have

$$
\triangle u=u_{t}+\int_{0}^{t} r(t-\tau) u_{\tau}(x, \tau) d \tau
$$

where $r(t)$ is resolvent of $k(t)$ and it satisfies the integral equation

$$
r(t)=k(t)+\int_{0}^{t} k(t-\tau) r(\tau) d \tau .
$$

In [42] it was shown the resolvent of $t^{-\alpha} / \Gamma(1-\alpha)$ is the function $(d / d t) E_{1-\alpha}\left(-t^{1-\alpha}\right)$. Computing the derivative of Mittag-Leffler function [43], we get

$$
\frac{d}{d t} E_{1-\alpha}\left(-t^{1-\alpha}\right)=t^{-\alpha} E_{1-\alpha, 1-\alpha}\left(-t^{1-\alpha}\right)
$$

We apply to both sides of (4.4) the Laplace and denoting by $K(s)$ and $R(s)$ the imagines of origins $k(t)$ and $r(t)$, respectively, obtain

$$
R(s)=K(s)+K(s) R(s) .
$$

In view of $K(s)=L\left[t^{-\alpha} E_{1-\alpha, 1-\alpha}\left(-t^{1-\alpha}\right)\right]=1 /\left(s^{1-\alpha}+1\right), \mathfrak{R}(s)>1$ (see [44]), from equality (4.5) we get

$$
R(s)=\frac{K(s)}{1-K(s)}=\frac{1}{s^{1-\alpha}}
$$

From here it follows

$$
L[R(s)]=L^{-1}\left[\frac{1}{s^{1-\alpha}}\right]=\frac{t^{-\alpha}}{\Gamma(1-\alpha)}=r(t) .
$$

Then, (4.3) yields (4.2).

Remark. Thus, the equation (4.1) with memory kernel $k(t)=t^{-\alpha} E_{1-\alpha, 1-\alpha}\left(-t^{1-\alpha}\right)$ describes the anomalously diffusive transport of solute in heterogeneous porous media.

From this remark it follows that the solution of equation (4.1) with conditions (1.2) can be given by formula $(3.1)$ for $f(x, t)=0$.

\section{Conclusions}

In applications, using the different types of a memory kernel $k(t)$ in equation (4.1) it can be described a wide variety of physical phenomena with memory effects. In this work, it is shown that $n$-dimensional anomalous diffusion equation (2.1) with $f(x, t)=0$ can be derived from the parabolic integro-differential equation (4.1) with memory kernel $t^{-\alpha} E_{1-\alpha, 1-\alpha}\left(-t^{1-\alpha}\right), \alpha \in(0,1)$. Based on the Laplace transform method to the time variable and Fourier transform to the spatial variable, the explicit solution of initial-boundary problem for anomalous diffusion equation is obtained. This solution includes the Prabhakar fractional integral and Fox H-functions. 


\section{References}

[1] V.M. Goloviznin, P.S. Kondratenko, L.V. Matveev et al. Anomalous diffusion of radionuclides of highly heterogeneous geological formations (Nauka, Moscow 2010) [in Russian].

[2] E. Abad, S. B. Yuste, K. Lindenberg, "Reaction-subdiffusion and reaction-superdiffusion equations for evanescent particles performing continuous-time random walks", Phys. Rev. E. $81(3), 031115$ (2010).

[3] A. V. Chechkin, R. Goreno, M. Sokolov, "Retarding subdiffusion and accelerating superdiffusion governed by distributed-order fractional diffusion equations", Phys. Rev. E. 66 (4), 046129 (2002).

[4] R. Metzler, W. G. Glockle, T. F. Nonnenmacher, "Fractional model equation for anomalous diffusion", Physica A. 211 (1), 13-24 (1994).

[5] R. Metzler, J. Klafter, "The random walk's guide to anomalous diffusion: a fractional dynamics approach", Phys. Rep. 339, 1-77 (2000).

[6] G. M. Zaslavsky, "Chaos, fractional kinetics, and anomalous transport", Phys. Rep. 371, 461-580 (2002).

[7] A. N. Bogolyubov, A. A. Potapov, S. Sh. Rikhviashvili, "The method of introducing fractional differentiation in classical electrodynamics", Bulletin of Moscow University. Ser. 3: Physics, astronomy. 4, 9-11 (2009).

[8] T. M. Atanackovic, S. Pilipovic, D. Zorica, "A diffusion-wave equation with two fractional derivatives of different order", J. Phys. A: Math. Theor. 40 (20), 5319-5333 (2007).

[9] Yu. Luchko, F. Mainardi, Yu. Povstenko, "Propagation speed of the maximum of the fundamental solution to the fractional diffusion-wave equation", Comput. Math. Appl. 66 (5), 774-784 (2013).

[10] F. Mainardi, "The time fractional diffusion-wave equation", Radiophys. Quantum Electron. 38 (1-2), 13-24 (1995).

[11] F. Mainardi, "Fractional relaxation-oscillation and fractional diffusion-wave phenomena", Chaos, Solitons and Fractals. 7 (9), 1461-1477 (1996).

[12] E. Barkai, "Fractional Fokker-Planck equation, solution, and application", Phys. Rev. E. 63 (4), 046118 (2001).

[13] A. V. Chechkin, J. Klafter, I. M. Sokolov, "Fractional Fokker-Planck equation for ultraslow kinetics", Europhys. Lett. 63 (3), 326-332 (2003). 
[14] R. Kazakevicius, J. Ruseckas, "Anomalous diffusion in nonhomogeneous media: power spectral density of signals generated by time-subordinated nonlinear Langevin equations", Physica A. 438, 210-222 (2015).

[15] R. Metzler, E. Barkai, J. Klafter, "Anomalous diffusion and relaxation close to thermal equilibrium: a fractional Fokker-Planck equation approach", Phys. Rev. Lett. 82 (18), 35633567 (1999).

[16] G. M. Zaslavsky, "Chaos, fractional kinetics, and anomalous transport", Phys. Rep. 371, 461-580 (2002).

[17] D. A. Benson, S. W. Wheatcraft, M. M. Meerschaert, "Application of a fractional advectiondispersion equation", Water Resour. Res. 36 (6), 1403-1412 (2000).

[18] D. Bolster, D. A. Benson, M. M. Meerschaert, B. Baeumer, "Mixing-driven equilibrium reactions in multidimensional fractional advection-dispersion systems", Physica A. 392 (10), 2513-2525 (2013).

[19] R. Schumer, D. A. Benson, M. M. Meerschaert, B. Baeumer, "Multiscaling fractional advection-dispersion equations and their solutions", Water Resour. Res. 39 (1), 1022 (2003).

[20] Y. Zhang, D. A. Benson, D. M. Reeves, "Time and space nonlocalities underlying fractionalderivative models: distinction and literature review of field applications", Adv. Water Resour. 32 (4), 561-581 (2009).

[21] Anomalous transport: foundations and applications, Ed. by R. Klages, G. Radons, I. M. Sokolov. (Willey-VCH, Berlin 2008).

[22] D. Del-Castillo-Negrete, B. A. Carreras, V. E. Lynch, "Front dynamics in reaction-diffusion systems with Levy flights: a fractional diffusion approach", Phys. Rev. Lett. 91 (1), 018302 (2003).

[23] R. K. Saxena, A. M. Mathai, H. J. Haubold, "Fractional reaction-diffusion equations ", Astrophys. Space Sci. 305 (3), 289-296 (2006).

[24] A. A. Kilbas, T. Pierantozzi, J. J. Trujillo, L. Vazquez, "On the solution of fractional evolution equations", J. Phys. A: Math. Gen. 37 (9), 3271-3283 (2004).

[25] A. A. Kilbas, H. M. Srivastava, J. J. Trujillo, Theory and applications of fractional differential equations (Amsterdam, Elsevier 2006).

[26] A. A. Kilbas, J. J. Trujillo, A. A. Voroshilov, "Cauchy-type problem for difussion-wave equation with the Riemann-Liouville partial derivative", Fract. Calc. Appl. Anal. 9 (2), 225-239 (2005).

[27] E. L. Shishkina, S. M. Sitnik Transmutations, singular and fractional differential equations with applications to mathematical physics, (Elsevier, Amsterdam, 2020). 
[28] A. N. Kochubei, S. D. Eidelman, "The fundamental solutions for the fractional diffusionwave equation", J. Differential Equations. 199 (2), 211-255 (2004).

[29] A. N. Kochubei, "Diffusion of fractional order", Differ. Equ. 26 (4), 485-492 (1990).

[30] Yu. Luchko, "Some uniqueness and existence results for the initial-boundary-value problems for the generalized time-fractional diffusion equation", Comput. Math. Appl. 59 (5), 1766$1772(2010)$.

[31] Yu. Luchko, "Anomalous diffusion: models, their analysis, and interpretation ", Advances in applied analysis / Ed. by S. Rogosin, A. Koroleva. Boston: Birkhauser Verlag, 115-145 (2012).

[32] F. Mainardi, "The fundamental solutions for the fractional diffusion-wave equation", Appl. Math. Lett. 9 (6), 25-28 (1996).

[33] F. Mainardi, Yu. Luchko, G. Pagnini, "The fundamental solution of the space-time fractional diffusion equation ", Fract. Calc. Appl. Anal. 4 (2), 153-192 (2001).

[34] A. Aghajani, Y. Jalilian, J. J. Trujillo, "On the existence of solutions of fractional integrodifferential equations", Fract. Calc. Appl. Anal. 15 (1), 44-69 (2012).

[35] R. Schumer, D. A. Benson, M. M. Meerschaert, and B. Baeumer, "Fractal mobile/immobile solute transport", Water Resour. Res., 39, 1-12 (2003).

[36] A. Giusti, I. Colombaro, R. Garra, at el., "Apractical guide to Prabhakar fractional calculus", Fract. Calc. Appl. Anal., 23 (1), 9-54 (2020).

[37] A. M. Mathai, R. K. Saxena, H. J. Haubold, The H-function: Theory and Application (Springer, Berlin/Heidelberg 2010).

[38] Z̆. Tomovski, T. Sandev, "Effects of a fractional friction with power-law memory kernel on string fibrations", Comput. Math. Appl. 62, 1554-1561 (2011).

[39] Z̆. Tomovski, R. Hilfer, H. M. Srivastava, "Fractional and operational calculus with generalized fractional derivatives operators and Mittag-Leffler type functions", Integral Transform. Spec. Funct. 21, 797-814 (2010).

[40] C. Li, W. H. Deng, X. Q. Shen, "Exact solutions and the asymptotic behaviors for the averaged generalized fractional elastic models", Commun. Theor. Phys. 62, 443-450 (2014).

[41] Y. Zao, F. Zao, "The analytical solution of parabolic Volterra integro-differential equations in the infinite domain", Entropy, 18 (344), 14 (2016).

[42] E. Hille, J. D. Tamarkin, "On the theory of linear integral equations", Ann. Math. 31, 479-528 (1930). 
[43] Yu. Luchko, "Initial-boundary-value problems for the one-dimensional time-fractional diffusion equation", Fract. Calc. Appl. Anal. 15 (1), 141-160 (2012).

[44] R. Gorenflo, F. Mainardi, "Fractional calculus: integral and differential equations of fractional order", Fractals and Fractional Calculus in Continuum Mechanics, Springer Verlag, Wien and New York, 223-276 (1997). 